\title{
Differential regulation of MAP kinase signalling by dual-specificity protein phosphatases
}

\author{
DM Owens and SM Keyse \\ Cancer Research UK Stress Response Laboratory, Biomedical Research Centre, Ninewells Hospital and Medical School, University \\ of Dundee, Dundee, Scotland, UK
}

The regulated dephosphorylation of mitogen-activated protein kinases (MAPKs) plays a key role in determining the magnitude and duration of kinase activation and hence the physiological outcome of signalling. In mammalian cells, an important component of this control is mediated by the differential expression and activities of a family of 10 dual-specificity (Thr/Tyr) MAPK phosphatases (MKPs). These enzymes share a common structure in which MAPK substrate recognition is determined by sequences within an amino-terminal non-catalytic domain whereas MAPK binding often leads to a conformational change within the $\mathrm{C}$-terminal catalytic domain resulting in increased enzyme activity. MKPs can either recognize and inactivate a single class of MAP kinase, as in the specific inactivation of extracellular signal regulated kinase (ERK) by the cytoplasmic phosphatase DUSP6/MKP-3 or can regulate more than one MAPK pathway as illustrated by the ability of DUSP1/MKP-1 to dephosphorylate ERK, c-Jun amino-terminal kinase and p38 in the cell nucleus. These properties, coupled with transcriptional regulation of $\mathrm{MKP}$ expression in response to stimuli that activate MAPK signalling, suggest a complex negative regulatory network in which individual MAPK activities can be subject to negative feedback control, but also raise the possibility that signalling through multiple MAPK pathways may be integrated at the level of regulation by MKPs.

Oncogene (2007) 26, 3203-3213. doi:10.1038/sj.onc.1210412

Keywords: DUSP; MKP; MAP kinase; phosphatase; signal transduction

\section{Introduction}

The mitogen-activated protein kinase (MAPK) cascades are conserved signal transduction pathways, which are activated in response to a diverse array of extracellular stimuli and can mediate both physiological and

Correspondence: Professor SM Keyse, Cancer Research UK Stress Response Laboratory, Biomedical Research Centre, Ninewells Hospital and Medical School, University of Dundee, Dundee DD1 9SY, Scotland, UK.

E-mail Stephen.Keyse@cancer.org.uk pathological responses in mammalian cells and tissues (Chang and Karin, 2001; Pearson et al., 2001; Dong et al., 2002; Wada and Penninger, 2004). These include cell proliferation, differentiation and transformation, stress responses, inflammation, growth arrest and apoptosis. The three major MAPK pathways are the extracellular signal regulated kinases (ERKs) 1 and 2 that are activated in response to growth factors and hormones and two distinct subfamilies of MAPK that respond to conditions of cellular stress. These are the cJun amino-terminal kinases (JNKs 1-3) and the p38 MAPKs $(\alpha, \beta, \gamma$ and $\delta)$. The core MAPK signalling module consists of a three-tier kinase cascade in which a MAPK kinase kinase (MKKK or MEKK) phosphorylates and activates a MAPK kinase (MKK or MEK). The latter is a dual-specificity protein kinase that phosphorylates both the threonine and tyrosine residues of the conserved T-X-Y motif and activates the MAPK itself (Marshall, 1994).

The physiological outcome of MAPK signalling depends on both the magnitude and duration of kinase activation (Marshall, 1995). In addition, the spatial distribution of MAPK activity can markedly alter the output of signalling, presumably by regulating access to substrates, dependent on subcellular localization (Ebisuya et al., 2005). The factors that influence the spatiotemporal regulation of MAPK signalling are both diverse and complex. They include the density and rates of internalization of cell surface receptors, the association of MAPK signalling components with scaffold proteins, and the balance between kinase activation and negative regulatory controls such as protein phosphatases (Volmat and Pouyssegur, 2001; Morrison and Davis, 2003; Murphy and Blenis, 2006). This latter class of proteins is now known to play an important role in the regulation of MAPK signalling (Keyse, 2000; Saxena and Mustelin, 2000). In particular, a family of 10 dualspecificity MAP kinase phosphatases (MKPs) acts to dephosphorylate and inactivate MAP kinase isoforms in mammalian cells. Certain MKPs are encoded by genes, which are transcriptionally upregulated by many stimuli that activate MAP kinase signalling. In addition, individual MKPs are able to differentially regulate MAPK isoforms. Thus, the MKPs form part of a complex negative regulatory network that acts to control MAPK pathway activities. This review explores the molecular mechanisms by which MKPs differentially 
Table 1 Nomenclature, key properties and physiological functions for dual-specificity MKPs

\begin{tabular}{|c|c|c|c|c|c|c|}
\hline Gene & $M K P$ & Trivial names & $\begin{array}{l}\text { Chromosomal } \\
\text { localization }\end{array}$ & $\begin{array}{l}\text { Subcellular } \\
\text { localization }\end{array}$ & $\begin{array}{l}\text { Substrate } \\
\text { preference }\end{array}$ & Physiological function(s) \\
\hline DUSP1 & MKP-1 & $\begin{array}{l}\text { CL100, erp, 3CH134, } \\
\text { hVH1 }\end{array}$ & $5 q 34$ & Nuclear & JNK, p38, ERK & $\begin{array}{l}\text { Negative regulator of immune } \\
\text { function. Protects mice from lethal } \\
\text { endotoxic shock. Plays a key role } \\
\text { in metabolic homoeostasis and } \\
\text { mediates resistance to cellular } \\
\text { stress in mouse fibroblasts. }\end{array}$ \\
\hline DUSP4 & MKP-2 & Typ1, Sty8, hVH2 & $8 \mathrm{p} 12-\mathrm{p} 11$ & Nuclear & JNK, p38, ERK & \\
\hline DUSP2 & & PAC-1 & $2 q 11$ & Nuclear & ERK, p38 & $\begin{array}{l}\text { Positive regulator of inflammatory } \\
\text { responses. Knockout mice are } \\
\text { resistant to immune inflammation }\end{array}$ \\
\hline DUSP5 & & hVH-3, B23 & $10 q 25$ & Nuclear & ERK & \\
\hline DUSP6 & MKP-3 & Pyst1, rVH6 & $12 q 22-q 23$ & Cytoplasmic & ERK & $\begin{array}{l}\text { Negative feedback regulator of } \\
\text { ERK } 2 \text { downstream of FGFR } \\
\text { signalling. }\end{array}$ \\
\hline DUSP7 & MKP-X & Pyst2, B59 & $3 \mathrm{p} 21$ & Cytoplasmic & ERK & \\
\hline DUSP9 & MKP-4 & Pyst3 & $\mathrm{Xq} 28$ & Cytoplasmic & ERK $>$ p38 & $\begin{array}{l}\text { Essential for placental develop- } \\
\text { ment and function (labyrinth } \\
\text { formation) }\end{array}$ \\
\hline DUSP8 & & M3/6, hVH5, HB5 & $11 \mathrm{p} 15.5$ & Cytoplasmic/nuclear & JNK, p38 & \\
\hline DUSP10 & MKP-5 & & $1 \mathrm{q} 41$ & Cytoplasmic/nuclear & JNK, p38 & $\begin{array}{l}\text { Functions in innate and adaptive } \\
\text { immunity }\end{array}$ \\
\hline DUSP16 & MKP-7 & & $12 \mathrm{p} 12$ & Cytoplasmic/nuclear & JNK, p38 & \\
\hline
\end{tabular}

Abbreviation: MKP, MAPK phosphatase.

recognize, bind and dephosphorylate MAP kinases and also discusses the evidence that has come from recent studies of MKP function which indicates that these enzymes play key roles in both the spatiotemporal regulation and integration of MAPK signalling.

\section{The dual-specificity MKPs}

The dual-specificity MKPs comprise a subfamily of 10 catalytically active proteins, all of which share a common structure comprising an N-terminal noncatalytic domain and a $\mathrm{C}$-terminal catalytic domain (Camps et al., 2000; Theodosiou and Ashworth, 2002). The non-catalytic domain contains two regions of homology with sequences flanking the active site of the Cdc25 cell cycle regulatory phosphatase and this reflects the fact that this domain of the MKPs and Cdc25 are both structurally related to the Rhodanese family of sulphotransferases (Keyse and Ginsburg, 1993; Bordo and Bork, 2002). The catalytic domain, which contains the characteristic cysteine-dependent protein tyrosine phosphatase active site sequence I/VHCXAGXXR, is related in sequence to the prototypic $\mathrm{VH}-1$ dualspecificity phosphatase encoded by vaccinia virus (Camps et al., 2000; Keyse, 2000). Based on gene structure, sequence similarity and subcellular localization, the 10 MKPs can be subdivided into three classes (Theodosiou and Ashworth, 2002). The first of these comprises DUSP1/MKP-1, DUSP2/PAC-1, DUSP4/ MKP-2 and DUSP5, all of which are inducible nuclear phosphatases. The second group comprises three closely related cytoplasmic phosphatases DUSP6/MKP-3, DUSP7/MKP-X and DUSP9/MKP-4 that preferentially inactivate ERK1 and ERK2. The third group comprises DUSP8, DUSP10/MKP-5 and DUSP16/ MKP-7 all of which show substrate selectivity towards the stress activated MAPK isoforms. Nomenclature and some basic properties of the MKPs are summarized in Table 1 .

Mechanisms of substrate recognition and catalysis The first MKP demonstrated to exhibit substrate specificity for a particular MAPK isoform was the cytoplasmic phosphatase DUSP6/MKP-3, which inactivates ERK $1 / 2$ in vitro and in vivo whereas displaying little or no activity towards either JNK or p38 (Groom et al., 1996; Muda et al., 1996). It was subsequently demonstrated that other MKPs such as DUSP8, DUSP10/MKP-5 and DUSP16/MKP-7 are able to selectively inactivate JNK and p38 but not ERK1/2 whereas others such as DUSP1/MKP-1 can regulate all three classes of MAPK (Muda et al., 1996; Tanoue et al., 1999, 2001b; Slack et al., 2001). The specific recognition and binding of ERK2 by DUSP6/MKP-3 is mediated by a conserved motif within the aminoterminal non-catalytic domain of the protein and is accompanied by a significant increase in the catalytic activity of DUSP6/MKP-3 in vitro (Camps et al., 1998; Muda et al., 1998). Based on these observations, catalytic activation was proposed as a general mechanism governing substrate selectivity for the MKPs (Camps et al., 2000). This idea was supported by subsequent work demonstrating that the activity of several other MKPs including DUSP1/MKP-1, DUSP4/ MKP-2, DUSP7/MKP-X and DUSP9/MKP-4 is also increased on binding to their MAPK substrates. This is particularly striking in the case of DUSP1/MKP-1, which undergoes catalytic activation on binding to ERK, JNK and p38, thus reflecting the ability of this phosphatase to recognize and inactivate all three major classes of MAPK in vivo (Slack et al., 2001). 
A major clue as to how the activity of MKPs is increased on binding to MAPKs came with the determination of the crystal structure of the catalytic domain of DUSP6/MKP-3 in the absence of substrate (Stewart et al., 1999). This revealed that key catalytic residues within DUSP6/MKP-3 display a distorted geometry. In particular, a loop containing an aspartic acid residue (Asp262), which performs an essential role as a general acid, is displaced such that it is $5.5 \AA$ away from the position of the equivalent residue in the related dual-specificity phosphatase VHR, and thus unlikely to participate in catalysis. This is consistent with a model in which ERK binding causes a conformational change within the catalytic domain of DUSP6/MKP-3 involving closure of the general acid loop over the active siteand positioning Asp262 for catalysis. This was confirmed by a combination of site directed mutagenesis and biochemical studies in which Asp262 was found to function as a general acid only in the presence of ERK2 (Stewart et al., 1999; Zhou and Zhang, 1999; Rigas et al., 2001). More recently, the structures of DUSP10/ MKP-5 and DUSP5 have also been obtained. Interestingly, unlike DUSP6/MKP-3, neither of these enzymes undergo catalytic activation on binding to their physiological MAPK substrates (Tanoue et al., 1999; Mandl et al., 2005) and in both structures the mobile loop containing the general acid residue is already positioned optimally for catalysis (Jeong et al., 2006a, b). This is consistent with biochemical studies indicating that the intrinsic catalytic activity of DUSP5 is actually higher than that of DUSP6/MKP-3 in the presence of ERK2 and that the general acid residue of DUSP5 is essential for this activity (Mandl et al., 2005). Thus, certain MKPs may rely only on MAPK recognition and binding in determining substrate selectivity and not secondary conformational change within their catalytic domain. This work leaves two important questions outstanding regarding substrate recognition and catalysis by these enzymes. Firstly, how do MKPs such as DUSP6/MKP3 and DUSP1/MKP-1 differentially recognize and bind to MAPK isoforms? Secondly, how does complex formation lead to conformational change within the catalytic domain of certain MKPs?

The specific recognition of ERK2 by DUSP6/MKP-3 requires a cluster of positively charged residues within the amino-terminal non-catalytic domain. Mutation of Arg64 and Arg65 within this kinase interaction motif (KIM) is sufficient to prevent dephosphorylation of ERK2 by DUSP6/MKP-3 (Nichols et al., 2000). This $\mathrm{KIM}$ is proposed to interact with negatively charged aspartic acid residues (Asp316 and 319) within a common docking (CD) site identified as an essential mediator of protein-protein interactions between MAPKs and their activators, regulators and substrates (Tanoue et al., 2000). It was quickly realized that the KIM was a conserved feature found within the aminoterminal domain of all dual-specificity MKPs (Figure 1) and attention focused on this region of the MKPs in determining the differential binding of these proteins to MAPK isoforms. One early clue that distinct binding determinants exist for ERK, JNK and p38 MAP kinases came with the finding that mutation of the conserved KIM within the nuclear phosphatase DUSP1/MKP-1 prevented binding to ERK and p38, but did not affect the ability of this phosphatase to recognize and inactivate JNK (Slack et al., 2001). Similar results were reported for the closely related enzyme DUSP4/MKP-2 (Chen et al., 2001).

In parallel, it was realized that the $\mathrm{CD}$ site within MAPKs is not the only determinant of specific interactions

\begin{tabular}{|c|c|c|}
\hline DUSP1/MKP-1 & 50-TIV $\underline{\underline{R R A K}} \mathrm{G}$ & AMGLEHIVP NAELRGRLLA-77 \\
\hline DUSP4/MKP-2 & $71-\mathrm{TIVRRRAK} \mathrm{G}$ & SVSLEQILPAEEEVRARLRS - 99 \\
\hline DUSP2/PAC1 & 53 -ALLRRRARGPPA & AAV LACLLP DRALRTRLVR-82 \\
\hline DUSP5/hVH-3 & 49 - SVVLRRARGG & AVSARYVLP DEAARARLLQ-77 \\
\hline DUSP6/MKP-3 & 60 -GIMLRRLQKG & NLPVRALFT RGEDRDRFTR-88 \\
\hline DUSP7/MKP-X & 47 - GLMLRRLRKG & NLPIRSIIPNHA DKERFAT-81 \\
\hline DUSP9/MKP-4 & 48 -ALLLRRLRRG & SLSVRALLP GPPLQP \\
\hline DUSP8/hVH-5 & 53 - KLVKRRLQQG & PAARSQVEA - 80 \\
\hline DUSP10/MKP-5 & $199-$ KISRRRLQQG & KITVLDLIS CREGKDSFKR-227 \\
\hline DUSP16/MKP-7 & 52 - KLMKRRLQQD & HSAKHKVDI - 79 \\
\hline
\end{tabular}

Figure 1 MKPs contain a modular MAPK binding surface. Sequence alignment of the conserved motifs, which are proposed to mediate MAP kinase recognition by the 10 human dual-specificity MKPs. Positively charged residues are in red, hydrophobic amino acids are in green and negatively charged residues are blue. The conserved arginine residues of the kinase interaction motif (KIM) are in bold and residues with confirmed roles in mediating MAPK-binding are underlined. The division of the MKPs into three distinct groups is based on overall sequence homology and indicated by the coloured backgrounds. The positions of the three conserved MAPK binding motifs are indicated. 


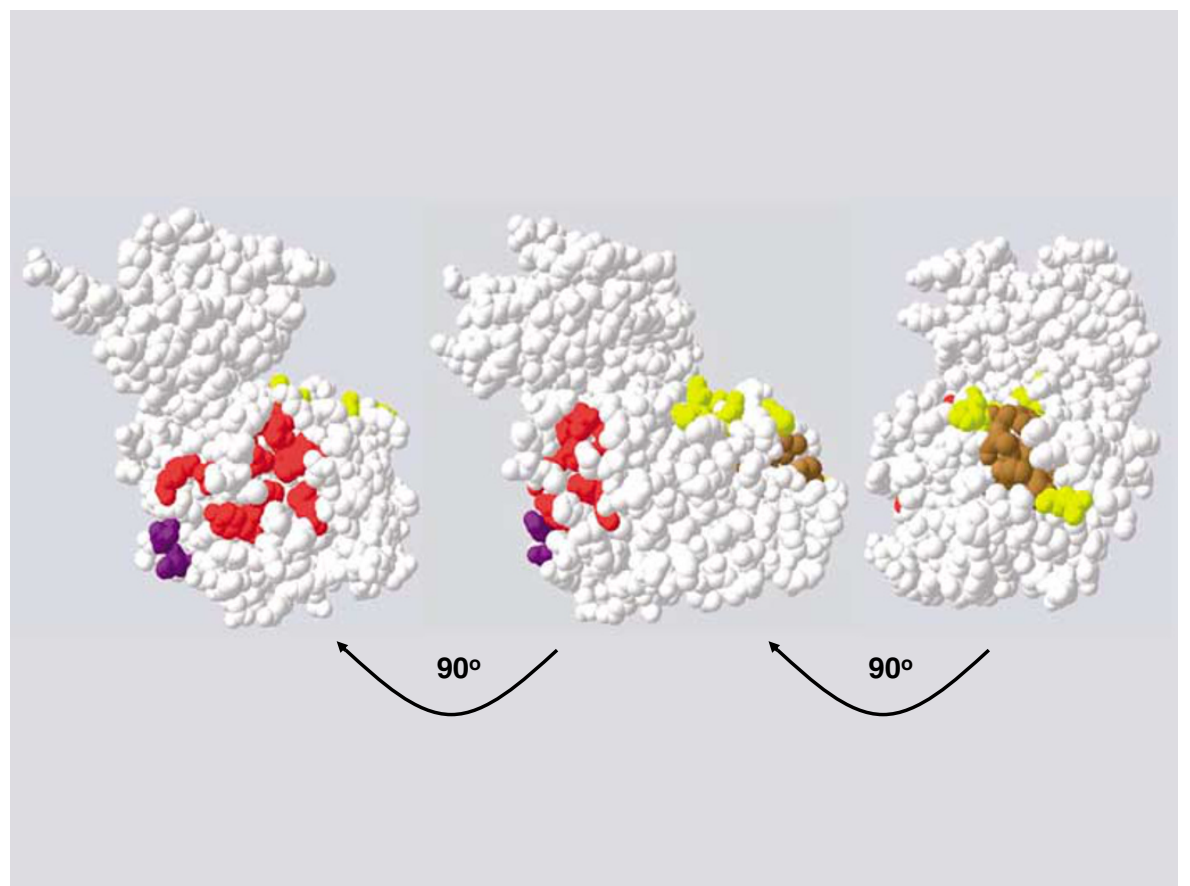

Figure 2 Surface representation of distinct docking domains on rat ERK2 (coordinates from www.rcsb.org), which are bound by the KIM and putative F-X-F-P motifs of MKP-3. Surface residues identified by reduced rates of hydrogen/deuterium (H/D) exchange and which have also been identified as important in binding to the KIM motif of MKP-3 are coloured red. The critical aspartic acid residues 316 and 319, which fall within this protected region and form primary contacts with Arg 64 and 65 within the KIM motif are coloured purple. On the opposite face of the larger C-terminal lobe of ERK2 residues that exhibit lower rates of H/D exchange in the presence of MKP-3 are coloured yellow and residues within this protected region which are essential for binding to the DEF (docking site for ERK, F/Y-X-F/Y-P) motifs of Elk-1 and c-Fos are coloured brown. A model is proposed in which the KIM of MKP-3 binds to the region containing Asp 316 and 319 whereas catalytic activation of MKP-3 is dependent on additional contacts between the putative F-X-F-P motif (FTAP) within the C-terminus of MKP-3 and the DEF docking domain.

with partner proteins. A second motif, the ED site, was identified within ERK2 and p38, which also contributes to the specificity of interactions between these MAPKs and binding partners including MKPs (Tanoue et al., 2001a). The solution of a crystal structure for p38 MAPK in complex with peptides derived from MEF2A, a p38 substrate, and MKK3, a p38 activator, further defined the interaction surface on the MAPKs by identifying an extended docking groove (DG) and also demonstrated that there were significant differences between interactions with substrates and activators (Chang et al., 2002).

With respect to the MKPs themselves, closer examination of the sequences adjacent to the KIM (Arg 53, 54 and 55) in DUSP1/MKP-1 revealed a second motif comprising Arg 72 and 74 surrounded by hydrophobic residues (Figure 1). Site-directed mutagenesis revealed that this motif was important for the ability of DUSP1/ MKP-1 to interact with JNK rather than p38 and, based on its positive charge, it was postulated to be the site of interaction with the negative residues of the ED site (Tanoue et al., 2002). A comparison of this extended docking region within DUSP1/MKP-1 with the corresponding sequences present in other MKPs led to the identification of a further area of conservation lying between the KIM and $\operatorname{Arg} 72 / 74$, which is abundant in hydrophobic residues (Figure 2). Mutation of either Met60 and Leu62 or Ile65 and Val66 to alanine caused loss of MKP-1 binding to both JNK and p38 indicating that this region is crucial for recognition of these kinases. Overall these studies suggest that the docking surface contained within the amino-termini of the MKPs is modular and that sequence variations with respect to position, number and species of charged amino acids within each module define the ability of MKPs to differentiate between and bind to specific MAPK isoforms (Tanoue et al., 2002).

Satisfyingly, a recent study in which a structure has been obtained for ERK2 in complex with the KIM peptide from DUSP6/MKP-3 confirms the primacy of the contacts between the basic residues of the KIM motif in mediating the interaction between these proteins (Liu et al., 2006). In particular, it identifies Arg65 of DUSP6/MKP-3 and Asp319 of ERK2 as providing the vast majority of side chain contacts in the ERK2-DUSP6/MKP-3 complex. This latter result is entirely consistent with previous biochemical and genetic studies where mutation of Arg65 causes a dramatic reduction in the affinity of DUSP6/MKP-3 for ERK2 and mutation of Asp319 to Asn in the sevenmaker gain of function mutant in Drosophila ERK/Rolled results in resistance of ERK to inactivation by protein phosphatases (Bott et al., 1994; Chu et al., 1996; Nichols et al., 2000). With respect to potential determinants of docking specificity, this study reinforces the concept that although MAP kinases may employ a 
common docking site for the KIM within MKPs, there are significant variations in the residues which are in direct contact with these motifs and which ensure binding specificity (Liu et al., 2006).

How then, once a MAPK substrate is engaged by an MKP, is binding linked to productive orientation of catalytic residues within the active site? To date, the structural basis of this phenomenon has been elusive as no crystal structure for a complete MKP has been solved and co-crystals of MAPK in complex with substrate proteins have proven extremely difficult to obtain. An NMR structure has been published for the isolated amino-terminal ERK-binding (EB) domain of DUSP6/MKP-3 (Farooq et al., 2001). This revealed the predicted structural homology between this domain of MKPs, the catalytic domain of the Cdc-25 cell cycleregulatory phosphatase and the rhodanese family of cysteine-dependent sulphotransferases and site-directed mutagenesis confirmed the importance of key residues within the KIM of DUSP6/MKP-3, for ERK2 binding (Farooq et al., 2001). In an attempt to probe the functional coupling between the EB and catalytic domains of DUSP6/MKP-3 NMR binding studies were performed using the catalytic domain of the related enzyme DUSP2/PAC-1, which is similar in structure to DUSP6/MKP-3 (Farooq et al., 2003). Although the binding affinity of the two recombinant domains is relatively weak when compared to the binding of the EB domain to ERK2, these experiments reveal that the EB domain may utilize overlapping regions for its interaction with either its C-terminal catalytic domain or ERK2 and that the contacts between the EB and catalytic domains involve both sequences near the active site and within the extreme C-terminus of DUSP6/ MKP-3. Overall these results, combined with the observation that the catalytic domain of DUSP6/ MKP-3 alone has very low phosphatase activity, would argue that the EB does not suppress the activity of DUSP6/MKP-3 by autoinhibition, but instead participates in a mechanism of substrate -induced activation (Farooq et al., 2001).

To gain further insights into the molecular basis of the specific ERK2 recognition by DUSP6/MKP-3, the technique of hydrogen/deuterium exchange mass spectrometry (H/DX-MS) has recently been employed to map the interface between these two proteins (Zhou et al., 2006). This technique relies on the propensity of amide protons within the peptide bonds of the protein to exchange with those of the solvent. If the latter is $\mathrm{D}_{2} \mathrm{O}$ rather than $\mathrm{H}_{2} \mathrm{O}$, the corresponding mass change can then be used to determine relative solvent accessibility. To analyse protein-protein interactions, the exchange reaction is carried out with the individual proteins and the complex and the regions of exchange compared. If a sequence is buried as a result of binding, the amides are protected, thus revealing the interaction interface (Mandell et al., 1998). Consistent with previous structural data, the KIM within DUSP6/MKP-3 (residues 64-76) displayed strong solvent protection as evidenced by a reduced rate of deuterium incorporation. In addition, residues between Tyr98 and Leu115, a segment which had not previously been implicated in mediating ERK2 binding, also displayed solvent protection. One drawback of $\mathrm{H} / \mathrm{DX}-\mathrm{MS}$ is an inability to distinguish between solvent inaccessibility owing to bona fide protein-protein interaction and that caused by conformational change within the protein structure. However site-directed mutagenesis coupled with in vitro binding studies using GST-pulldown assays confirms that substitution of either Leu97 or Asn 106 abrogates ERK2 binding, indicating a role for this sequence in substrate recognition (Zhou et al., 2006).

With regard to the $\mathrm{C}$-terminal catalytic domain of DUSP6/MKP-3, a major surprise was the extent of the reduction in $\mathrm{H} / \mathrm{D}$ exchange rates throughout this region. This is unlikely to reflect direct protein contact, and mutagenesis of a number of surface exposed residues within this domain confirms that they play no role in mediating interactions with ERK2 (Zhou et al., 2006). Thus, it is more likely that reduced rates of exchange reflect an overall decrease in protein dynamics and flexibility. This is consistent with an ERK-induced conformational change. Finally, a putative F-X-F-P ERK docking motif (FTAP, residues 364-367) within the C-terminal tail of DUSP6/MKP-3 also displayed reduced deuterium incorporation. This sequence was not present in the truncated domain used for structural determination (Stewart et al., 1999), but biochemical studies had previously indicated that although this motif was not essential for ERK binding, it was required for ERK-induced activation of DUSP6/MKP-3 (Zhou et al., 2001).

With respect to ERK2 itself, contacts with DUSP6/ MKP-3 were identified, which correspond to the CD domain and DG and are thus are in agreement with both structural data and mutagenesis experiments (Figure 2). In addition, strong solvent protection was observed in three segments Phe166-Leu198, Leu220Glu248 and Ile253-Leu262. These sequences include the T-X-Y signature phosphorylation motif (residues 183$185)$ in the activation loop of ERK2 and also sequences within the MAP kinase insert (Zhou et al., 2006). Interestingly, this latter region was also identified as essential for the binding of proteins containing DEF (docking site for ERK, $\underline{F} / \mathrm{Y}-\mathrm{X}-\mathrm{F} / \mathrm{Y}-\mathrm{P}$ ) motifs such as the Ets family transcription factor Elk-1 and c-Fos (Dimitri et al., 2005) and these peptides were also identified as exhibiting reduced $\mathrm{H} / \mathrm{D}$ exchange in an independent study using an F-X-F-P-containing peptide derived from the Elk-1 transcription factor (Lee et al., 2004). Mutation of residues in the DEF domain binding pocket of ERK2 (Lys257 and Tyr261 to Ala) did not affect binding to DUSP6/MKP-3, but did cause a reduction in catalytic activation of the phosphatase by as much as $85 \%$. Taken together, these results allow the formulation of a model in which a primary interaction between the KIM containing peptide of DUSP6/MKP-3 and the CD domain of ERK coupled with additional contacts involving the FTAP motif of DUSP6/MKP-3 and the ERK DEF-binding pocket combine to effect allosteric activation of the phosphatase (Figure 2) (Zhou et al., 2006). Although these studies offer a tantalizing glimpse 
of the possible mechanisms with respect to MKP-3 substrate recognition and catalytic activation, it remains to be demonstrated that this bipartite model of binding is more generally applicable to the substrate interactions of other MKPs. The final resolution of these questions may only be possible when structural information for complexes between MAPKs and MKPs becomes available.

\section{MKPS play essential roles in a wide range of physiological processes}

The ability of dual specificity MKPs to differentially bind and inactivate MAPK isoforms has the potential to significantly modify the biological outcome of signalling. However, despite our knowledge of the mechanisms underlying substrate recognition and catalysis, the physiological roles of MKPs are largely unexplored. An initial genetic experiment in which the murine DUSP1/ MKP-1 gene was knocked out failed to detect any obvious phenotype in either the null animals or cells derived from them (Dorfman et al., 1996). These results were interpreted, as indicating that either DUSP1/ MKP-1 did not play a significant role in regulating MAPK activity or there is a significant degree of functional redundancy within the MKP subfamily. However, reappraisal of the DUSP1/MKP-1 null mice combined with additional mouse knockouts and developmental studies have begun to demonstrate interesting and often unexpected roles for the MKPs in regulating a number of physiological processes. These include immune and metabolic function, the regulation of stress responses and development.

\section{MKPs and the regulation of immune function}

Mouse knockout experiments have now demonstrated that three MKPs play essential non-redundant roles in regulating immune function. These are DUSP1/MKP-2, DUSP2/PAC-1 and DUSP10/MKP-5. Although mice lacking either DUSP1/MKP-1 or DUSP10/MKP-5 show no developmental defects with respect to the immune system, both animals have defects in innate and adaptive immunity. In the case of DUSP1/MKP-1, null animals are sensitized to lethal endotoxic shock and exhibit a marked increase in incidence and severity of experimentally induced autoimmune arthritis (Chi et al., 2006; Hammer et al., 2006; Salojin et al., 2006; Zhao et al., 2006). This is accompanied by the overproduction of both pro- and anti-inflammatory cytokines (Figure 3a). At the cellular level, macrophages from the null animals show increased p38 and JNK MAPK activities in response to bacterial lipopolysaccharide (LPS). With respect to cytokine production, at early times after exposure to LPS DUSP1/MKP-1 null macrophages produce increased levels of the proinflammatory cytokine tumour necrosis factor (TNF) when compared with wild-type cells. In contrast, production of the anti-inflammatory cytokine interleukin (IL)-10 is both immediate and prolonged in duration (Chi et al., 2006).

The expression of DUSP10/MKP-5 is strongly induced in macrophages exposed to LPS. In the T-cell compartment, expression of DUSP10/MKP-5 is constitutive in naïve cells but downregulated following T-cell activation. In DUSP10/MKP-5 null animals, elevated JNK activities are seen in both T-cells and also in macrophages exposed to LPS whereas p38 activity appears to be unaffected (Zhang et al., 2004). Thus, the major role of DUSP10/MKP-5 appears to be the regulation of JNK activity during innate or adaptive immune responses. As was seen in the DUSP1/MKP-1 null mice, macrophages lacking DUSP10/MKP-5 overproduce proinflammatory cytokines when exposed to LPS (Figure 3b). However, in the T-cell compartment

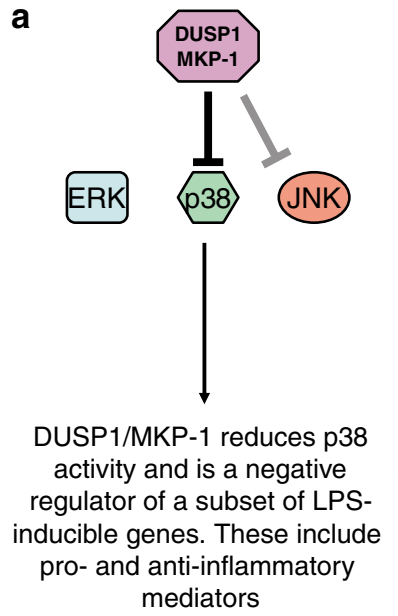

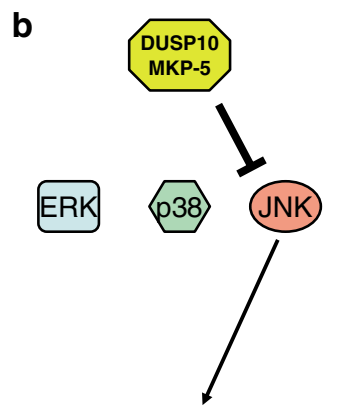

DUSP10/MKP-5 reduces JNK activity and is a negative regulator of cytokine genes including TNF and IL-6

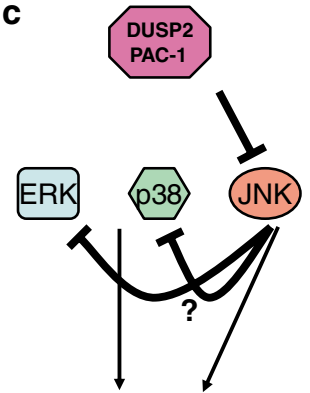

DUSP2/PAC- 1 reduces JNK activity thus alleviating JNKmediated repression of ERK and p38 and acting as a positive effector of inflammatory Gene expression

Figure 3 Regulation of MAPK activities by MKPs in immune effector cells. (a) DUSP1/MKP-1 predominantly inactivates nuclear p38 MAPK in mouse macrophages and acts as a negative regulator of a subset of genes in response to LPS challenge. (b) DUSP10/ MKP-5 appears to act mainly via regulation of JNK activity and is a negative regulator of TNF and IL-6 synthesis in macrophages. (c) The mouse knockout of DUSP2/PAC-1 reveals unexpected effects on MAPK activities in mouse macrophages. JNK activity is elevated whereas ERK and p38 activities are reduced by an as yet unknown mechanism of JNK-dependent crosstalk. This leads to a deficit in cytokine synthesis and provides evidence that DUSP2/PAC-1 is a positive regulator of immune function. 
the picture is more complex. Firstly antigen-presenting cells from DUSP10/MKP-5 null animals exhibit increased T-cell priming activity following exposure to LPS. However, despite the overproduction of both T-helper cell 1 and T-helper cell 2 (Th1 and Th2) cytokines, T-cell proliferation is impaired. The latter effect probably accounts for the resistance of DUSP10/ MKP-5 null mice to experimental autoimmune encephalitis following injection of myelin oligodendrocyte glycoprotein. A positive role for DUSP10/MKP-5 in T-cell expansion is consistent with previous studies indicating that JNK itself plays an inhibitory role in T-cell growth (Dong et al., 1998). In terms of T-cellmediated immunity, DUSP10/MKP-5 null mice show little difference in primary response to infection with lymphocytic choriomeningitis virus (LCV) when compared to wild-type animals. However, secondary exposure to LCV causes significant immune-mediated lethality among DUSP10/MKP-5 null mice. This is most probably associated with the high levels of serum TNF seen after viral challenge (Zhang et al., 2004).

The third MKP, which plays a key role in regulating immune function, is DUSP2/PAC-1. This gene was originally characterized as encoding a mitogen-induced phosphatase in human $\mathrm{T}$ cells and its induction appears to dependent on the activation of ERK1 and 2, suggesting that it functions as part of a negative feedback control of MAPK activity (Rohan et al., 1993; Grumont et al., 1996). With respect to substrate specificity, PAC-1 can dephosphorylate ERK and p38 in vitro and in vivo, but only ERK appears to cause catalytic activation of DUSP2/PAC-1 in vitro (Chu et al., 1996; Zhang et al., 2005). DUSP2/PAC1 was also identified as a p53 target gene in fibroblasts following oxidative stress and small interfering RNAmediated knockdown of DUSP2/PAC1 correlated with increased ERK activation and protected cells from apoptosis (Yin et al., 2003). Despite this apparent specificity for ERK and p38, the results of a recent gene knockout for DUSP2/PAC-1 have revealed considerable complexity in the regulation of MAPK activities by this phosphatase and uncovered an unexpected role for DUSP2/PAC-1 as a positive regulator of inflammatory responses (Jeffrey et al., 2006). Firstly, DUSP2/PAC-1 null mice display much reduced levels of inflammation in an autoimmune model of rheumatoid arthritis where disease progression is dependent on effector leucocytes such as macrophages mast cells and neutrophils. At the cellular level, LPS-stimulated DUSP2/PAC-1 null macrophages also produce much lower levels of proinflammatory cytokines and chemokines when compared with wild-type cells (Figure 3c). Furthermore, despite the expectation that DUSP2/PAC-1 loss would result in increased activation of ERK and possibly p38, the deficit in synthesis of inflammatory mediators is accompanied by a decrease in ERK and p38 activities and an increase in JNK activation (Jeffrey et al., 2006). The downregulation of ERK appears to be the result of crosstalk with JNK as pharmacological inhibition of JNK increases ERK activity.
What then is the mechanistic basis for the nonredundant functions of these MKPs in immune regulation? In the case of DUSP1/MKP-1 and DUSP10/ MKP-5, a key factor may be the extent to which these two MKPs influence the activities of different MAPK pathways. The major target of MKP-1, at least in macrophages, appears to be p38. Consistent with this, the overproduction of cytokines and inflammatory mediators seen in DUSP1/MKP-1 null macrophages is suppressed by SB203580, a specific p38 inhibitor of p38 (Chi et al., 2006; Hammer et al., 2006). In contrast, loss of DUSP10/MKP-5 results in increased JNK activity with little of no change seen in p38 activation (Zhang et al., 2004). Another key factor may be the spatial restriction of MKP activity. Unlike DUSP10/MKP-5, which is found in both the nucleus and the cytoplasm (Tanoue et al., 1999), DUSP1/MKP-1 is a nuclear enzyme and may act differentially to regulate nuclear but not cytoplasmic MAPK functions (Wu et al., 2005). Support for the latter idea has come from a recent study, again using null mice, in which DUSP1/MKP-1 was identified as a key regulator of metabolic homoestasis (Wu et al., 2006). Mice lacking DUSP1/MKP-1 do not gain weight when fed either normal chow or on a highfat diet when compared with wild-type animals. Resistance to diet-induced obesity is accompanied by complex changes in the activities of ERK, JNK and p38 in insulin responsive tissues. Elevated JNK and p38 activation is seen in liver, white adipose tissue (WAT) and skeletal muscle, whereas increased ERK activity is seen in WAT and muscle, but not in liver. In the latter tissue, there is very good evidence that phosphorylation of nuclear JNK targets such as c-Jun is increased, whereas the phosphorylation of insulin receptor substrate-1, a cytoplasmic target of $\mathrm{JNK}$, is unaffected (Wu et al., 2006). Thus, the spatial restriction of DUSP1/ MKP-1 activity, as well as its ability to differentially regulate the three major MAPK pathways, may underpin the unique aspects of the DUSP1/MKP-1 knockout with respect to immune and metabolic functions.

Although the effects of removing either DUSP1/ MKP-1 or DUSP10/MKP-5 can be rationalized with previous results of biochemical and transfection experiments in terms of MAPK substrate preferences, the changes in MAPK activities seen on DUSP2/PAC-1 deletion are unexpected. Expression studies have demonstrated that DUSP2/PAC-1 inactivates ERK and p38 but not JNK in vitro and in vivo (Chu et al., 1996). Further studies of DUSP2/PAC-1 function in immune effector cells will be required to elucidate the mechanism by which this MKP is able to target JNK and also the exact nature of the interactions which lead to suppression of ERK and p38 activities.

\section{Cytoplasmic ERK-specific MKPs play essential roles during early development}

The closely related phosphatases DUSP6/MKP-3, DUSP7/MKP-X and DUSP9/MKP-4 constitute a subgroup of cytoplasmic MKPs that exhibit substrate selectivity towards ERK1 and 2 MAPKs (Table 1). 
DUSP9/MKP-4 was the first member of this group of MKPs to be analysed by gene deletion. Mice lacking DUSP9/MKP-4 die in utero from placental insufficiency, which is secondary to a failure of labyrinth development (Christie et al., 2005). Based on biochemical evidence and transfection experiments, the most likely substrates for DUSP9/MKP-4 in vivo are ERK1 and 2 and/or p38 MAPK (Dickinson et al., 2002b). However, no significant abnormalities in the phosphorylation levels of either of these MAPKs were observed in null placental tissues when compared with wild type (Christie et al., 2005). This may indicate that these enzymes are not targeted by DUSP9/MKP-4. Alternatively, the signalling abnormality may either be very transient or of such low magnitude to make its detection difficult. Interestingly, deletion of either $\mathrm{p} 38 \alpha$ or MEK 1 (the upstream activator of ERK) leads to placental defects that are almost identical to those seen on deletion of DUSP9/MKP-4 (Giroux et al., 1999; Adams et al., 2000), a possible indication that these MAPKs may indeed be targets for this phosphatase. Although necessary for placental development and function, tetraploid rescue experiments demonstrate that DUSP9/MKP-4 is entirely dispensable for normal embryogenesis. Furthermore, organs such as liver, kidney and testis, in which DUSP9/MKP-4 is expressed either during development or in the adult, exhibit normal tissue morphology in mice lacking DUSP9/ MKP-4 (Christie et al., 2005).

The ERK-specific phosphatase DUSP6/MKP-3 is by far the most studied of this group of three cytoplasmic MKPs. The first clue as to a physiological function for DUSP6/MKP-3 came with the observation that it is expressed at many sites of fibroblast growth factor (FGF) signalling during early mouse development (Dickinson et al., 2002a). Subsequent studies in the developing limbs and nervous tissues in chicken embryos demonstrated that DUSP6/MKP-3 expression was mediated by FGFs as a component of a negative feedback control to regulate levels of activated ERK MAPK in these tissues (Eblaghie et al., 2003; Kawakami et al., 2003). There is currently some confusion as to which of the intracellular signalling pathways that lie downstream of the FGF-receptor is responsible for mediating DUSP6/MKP-3 expression, with evidence supporting a role for either ERK MAPK or PI3-kinase signalling in this process (Eblaghie et al., 2003; Kawakami et al., 2003; Tsang et al., 2004; Gomez et al., 2005; Smith et al., 2005). Most of these data were obtained using different pharmacological inhibitors of the ERK pathway and this may explain some of the apparently contradictory results (Smith et al., 2006).

Overexpression of DUSP6/MKP-3 in either the chicken limb or neural plate causes abnormalities in these tissues, whereas either overexpression or morpholino-mediated knockdown of DUSP6/MKP-3 in zebrafish embryos causes profound defects in dorsoventral patterning (Eblaghie et al., 2003; Tsang et al., 2004). This is presumably because DUSP6/MKP-3 acts to maintain FGF-mediated ERK activities at levels that are permissible for normal tissue growth and development.
Support for such a role has now come with, the result of a mouse knockout for DUSP6/MKP-3 (Li et al., 2007). Surprisingly, given the severity of the defects seen following morpholino-mediated knockdown of DUSP6/MKP-3 in Zebrafish embryos, mouse embryos lacking this phosphatase do not present with any obvious abnormalities. However, embryos lacking DUSP6/MKP-3 do show evidence of increased levels of phosphorylated ERK2 in the limb buds and present with a variably penetrant postnatal lethality, skeletal dwarfism, premature fusion of the cranial sutures (craniostynostoses) and hearing loss (Li et al., 2007). All of these features are consistent with increased activity of FGF receptor (FGFR)-mediated signalling pathways although they do not precisely mimic the results of any particular FGF receptor gain of function mutant. This together with the incomplete penetrance of the phenotype suggests that DUSP6/MKP-3 may function downstream of more than one FGFR and that there may also be some degree of redundancy between DUSP6/MKP-3 and other negative regulators of FGF signalling, including the closely related phosphatases DUSP7/MKP-X and DUSP9/MKP-4 (Li et al., 2007).

\section{Conclusions}

With respect to the differential recognition and dephosphorylation of MAPKs by dual-specificity MKPs, we understand some details of the molecular mechanisms involved, but questions still remain. Principal among these are the full extent of the binding interactions between MKP and MAPK, which must determine substrate selectivity, and the precise way in which conformational change within the catalytic domain of MKPs occurs, leading to increased activity. In addition, MKPs such as DUSP5 and DUSP10/MKP-5 do not undergo catalytic activation in vitro on binding to substrate MAPKs, indicating that these enzymes rely on specific protein-protein interactions alone to determine substrate selectivity. It could be argued that assaying catalytic activation using the chromogenic substrate para-nitrophenyl phosphate is not physiological and could be misleading. However, the crystal structures of the catalytic domains of both DUSP5 and DUSP10/ MKP-5 show that, unlike DUSP6/MKP-3, these enzymes adopt a catalytically favourable geometry in the absence of substrate. Why certain MKPs require secondary conformational change on substrate binding to be fully active, whereas others do not, is unclear.

How the differential activity of MKPs is expressed in physiological terms is becoming more apparent with the generation of mice lacking individual MKP genes. Thus far, it is clear that the ability of MKPs such as DUSP1/ MKP-1 to regulate at least three classes of MAPK in mammalian cells is critical to its ability to perform distinct and important functions in the immune system and in the regulation of metabolic homoeostasis. This suggests that signalling through multiple MAPK pathways may be integrated at the level of MKP expression. 
Furthermore, it is also clear that the spatial restriction of MKP activity may be critical in determining outcome as exemplified by the ability of DUSP1/MKP-1 to inactivate the nuclear as opposed to cytoplasmic pool of JNK. The recent finding that both DUSP8 and DUSP16/MKP-7 can bind to the JNK scaffold proteins JNK-interacting protein- 1 and $\beta$-arrestin 2 indicates that the spatial restriction of the activities of MKPs will be an increasingly important component of their function (Willoughby et al., 2003; Willoughby and Collins, 2005). Finally, the phenotypes observed following ablation of certain MKPs such as DUSP1/MKP-1, DUSP10/MKP-5 and DUSP6/MKP-3 are consistent

\section{References}

Adams RH, Porras A, Alonso G, Jones M, Vintersten K, Panelli S et al. (2000). Essential role of p38alpha MAP kinase in placental but not embryonic cardiovascular development. Mol Cell 6: 109-116.

Bordo D, Bork P. (2002). The rhodanese/Cde25 phosphatase superfamily. Sequence-Structure-Function relations. $E M B O$ Rep 3: 741-746.

Bott CM, Thorneycroft SG, Marshall CJ. (1994). The sevenmaker gain-of-function mutation in p42 MAP kinase leads to enhanced signalling and reduced sensitivity to dual specificity phosphatase action. FEBS Lett 352: 201-205.

Camps M, Nichols A, Arkinstall S. (2000). Dual specificity phosphatases: a gene family for control of MAP kinase function. FASEB $J$ 14: 6-16.

Camps M, Nichols A, Gillieron C, Antonsson B, Muda M, Chabert C et al. (1998). Catalytic activation of the phosphatase MKP-3 by ERK2 mitogen-activated protein kinase. Science 280: 1262-1265.

Chang CI, Xu BE, Akella R, Cobb MH, Goldsmith EJ. (2002). Crystal structures of MAP kinase p38 complexed to the docking sites on its nuclear substrate MEF2A and activator MKK3b. Mol Cell 9: 1241-1249.

Chang L, Karin M. (2001). Mammalian MAP kinase signalling cascades. Nature 410: 37-40.

Chen P, Hutter D, Yang X, Gorospe M, Davis RJ, Liu Y. (2001). Discordance between the binding affinity of mitogen-activated protein kinase subfamily members for MAP kinase phosphatase-2 and their ability to activate the phosphatase catalytically. J Biol Chem 276: 29440-29449.

Chi H, Barry SP, Roth RJ, Wu JJ, Jones EA, Bennett AM et al. (2006). Dynamic regulation of pro- and antiinflammatory cytokines by MAPK phosphatase 1 (MKP1) in innate immune responses. Proc Natl Acad Sci USA 103: $2274-2279$

Christie GR, Williams DJ, Macisaac F, Dickinson RJ, Rosewell I, Keyse SM. (2005). The dual-specificity protein phosphatase DUSP9/MKP-4 is essential for placental function but is not required for normal embryonic development. Mol Cell Biol 25: 8323-8333.

Chu Y, Solski PA, Khosravi-Far R, Der CJ, Kelly K. (1996). The mitogen-activated protein kinase phosphatases PAC1, MKP-1, and MKP-2 have unique substrate specificities and reduced activity in vivo toward the ERK2 sevenmaker mutation. J Biol Chem 271: 6497-6501.

Dickinson RJ, Eblaghie MC, Keyse SM, Morriss-Kay GM. (2002a). Expression of the ERK-specific MAP kinase phosphatase PYST1/MKP3 in mouse embryos during morphogenesis and early organogenesis. Mech Dev 113: 193-196. with previous biochemical and overexpression studies in terms of MAPK regulation. In contrast, studies of DUSP9/MKP-4 null animals have not yet led to an unequivocal linkage between the activity of this MKP and target MAPK pathways. In the case of mice lacking DUSP2/PAC-1, the changes seen in the activities of individual MAPKs are unexpected given the results of previous work to determine the cellular targets for this phosphatase. Future studies will be directed towards the resolution of these questions and when combined with the generation of additional mouse knockout models should lead to a comprehensive understanding of MKP function in both normal physiology and disease.

Dickinson RJ, Williams DJ, Slack DN, Williamson J, Seternes OM, Keyse SM. (2002b). Characterization of a murine gene encoding a developmentally regulated cytoplasmic dualspecificity mitogen-activated protein kinase phosphatase. Biochem J 364: 145-155.

Dimitri CA, Dowdle W, MacKeigan JP, Blenis J, Murphy LO. (2005). Spatially separate docking sites on ERK2 regulate distinct signaling events in vivo. Curr Biol 15: 1319-1324.

Dong C, Davis RJ, Flavell RA. (2002). MAP kinases in the immune response. Annu Rev Immunol 20: 55-72.

Dong C, Yang DD, Wysk M, Whitmarsh AJ, Davis RJ, Flavell RA. (1998). Defective T cell differentiation in the absence of Jnk1. Science 282: 2092-2095.

Dorfman K, Carrasco D, Gruda M, Ryan C, Lira SA, Bravo R. (1996). Disruption of the erp/mkp-1 gene does not affect mouse development: normal MAP kinase activity in ERP/ MKP-1-deficient fibroblasts. Oncogene 13: 925-931.

Ebisuya M, Kondoh K, Nishida E. (2005). The duration, magnitude and compartmentalization of ERK MAP kinase activity: mechanisms for providing signaling specificity. J Cell Sci 118: 2997-3002.

Eblaghie MC, Lunn JS, Dickinson RJ, Munsterberg AE, SanzEzquerro JJ, Farrell ER et al. (2003). Negative feedback regulation of FGF signaling levels by Pyst1/MKP3 in chick embryos. Curr Biol 13: 1009-1018.

Farooq A, Chaturvedi G, Mujtaba S, Plotnikova O, Zeng L, Dhalluin C et al. (2001). Solution structure of ERK2 binding domain of MAPK phosphatase MKP-3: structural insights into MKP-3 activation by ERK2. Mol Cell 7: 387-399.

Farooq A, Plotnikova O, Chaturvedi G, Yan S, Zeng L, Zhang Q et al. (2003). Solution structure of the MAPK phosphatase PAC-1 catalytic domain. Insights into substrate-induced enzymatic activation of MKP. Structure 11: $155-164$.

Giroux S, Tremblay M, Bernard D, Cardin-Girard JF, Aubry S, Larouche L et al. (1999). Embryonic death of Mek1deficient mice reveals a role for this kinase in angiogenesis in the labyrinthine region of the placenta. Curr Biol 9: 369-372.

Gomez AR, Lopez-Varea A, Molnar C, de la Calle-Mustienes E, Ruiz-Gomez M, Gomez-Skarmeta JL et al. (2005). Conserved cross-interactions in Drosophila and Xenopus between Ras/MAPK signaling and the dual-specificity phosphatase MKP3. Dev Dyn 232: 695-708.

Groom LA, Sneddon AA, Alessi DR, Dowd S, Keyse SM. (1996). Differential regulation of the MAP, SAP and RK/ p38 kinases by Pyst1, a novel cytosolic dual-specificity phosphatase. EMBO J 15: 3621-3632. 
Grumont RJ, Rasko JE, Strasser A, Gerondakis S. (1996). Activation of the mitogen-activated protein kinase pathway induces transcription of the PAC-1 phosphatase gene. Mol Cell Biol 16: 2913-2921.

Hammer M, Mages J, Dietrich H, Servatius A, Howells N, Cato AC et al. (2006). Dual specificity phosphatase 1 (DUSP1) regulates a subset of LPS-induced genes and protects mice from lethal endotoxin shock. J Exp Med 203: 15-20.

Jeffrey KL, Brummer T, Rolph MS, Liu SM, Callejas NA, Grumont RJ et al. (2006). Positive regulation of immune cell function and inflammatory responses by phosphatase PAC-1. Nat Immunol 7: 274-283.

Jeong DG, Cho YH, Yoon TS, Kim JH, Ryu SE, Kim SJ. (2006a). Crystal structure of the catalytic domain of human DUSP5, a dual specificity MAP kinase protein phosphatase. Proteins 66: 253-258.

Jeong DG, Yoon TS, Kim JH, Shim MY, Jung SK, Son JH et al. (2006b). Crystal structure of the catalytic domain of human MAP kinase phosphatase 5: structural insight into constitutively active phosphatase. J Mol Biol 360: 946-955.

Kawakami Y, Rodriguez-Leon J, Koth CM, Buscher D, Itoh T, Raya A et al. (2003). MKP3 mediates the cellular response to FGF8 signalling in the vertebrate limb. Nat Cell Biol 5: 513-519.

Keyse SM. (2000). Protein phosphatases and the regulation of mitogen-activated protein kinase signalling. Curr Opin Cell Biol 12: 186-192.

Keyse SM, Ginsburg M. (1993). Amino acid sequence similarity between CL100, a dual-specificity MAP kinase phosphatase and cdc25. Trends Biochem Sci 18: 377-378.

Lee T, Hoofnagle AN, Kabuyama Y, Stroud J, Min X, Goldsmith EJ et al. (2004). Docking motif interactions in MAP kinases revealed by hydrogen exchange mass spectrometry. Mol Cell 14: 43-55.

Li C, Scott DA, Hatch E, Tian X, Mansour SL. (2007). Dusp6 (Mkp3) is a negative feedback regulator of FGF-stimulated ERK signaling during mouse development. Development 134: $167-176$.

Liu S, Sun JP, Zhou B, Zhang ZY. (2006). Structural basis of docking interactions between ERK2 and MAP kinase phosphatase 3. Proc Natl Acad Sci USA 103: 5326-5331.

Mandell JG, Falick AM, Komives EA. (1998). Identification of protein-protein interfaces by decreased amide proton solvent accessibility. Proc Natl Acad Sci USA 95: 14705-14710.

Mandl M, Slack DN, Keyse SM. (2005). Specific inactivation and nuclear anchoring of extracellular signal-regulated kinase 2 by the inducible dual-specificity protein phosphatase DUSP5. Mol Cell Biol 25: 1830-1845.

Marshall CJ. (1994). MAP kinase kinase kinase, MAP kinase kinase and MAP kinase. Curr Opin Genet Dev 4: 82-89.

Marshall CJ. (1995). Specificity of receptor tyrosine kinase signaling: transient versus sustained extracellular signalregulated kinase activation. Cell 80: 179-185.

Morrison DK, Davis RJ. (2003). Regulation of MAP kinase signaling modules by scaffold proteins in mammals. Annu Rev Cell Dev Biol 19: 91-118.

Muda M, Theodosiou A, Gillieron C, Smith A, Chabert C, Camps $\mathrm{M}$ et al. (1998). The mitogen-activated protein kinase phosphatase-3 N-terminal noncatalytic region is responsible for tight substrate binding and enzymatic specificity. J Biol Chem 273: 9323-9329.

Muda M, Theodosiou A, Rodrigues N, Boschert U, Camps M, Gillieron C et al. (1996). The dual specificity phosphatases M3/6 and MKP-3 are highly selective for inactivation of distinct mitogen-activated protein kinases. J Biol Chem 271: 27205-27208.

Murphy LO, Blenis J. (2006). MAPK signal specificity: the right place at the right time. Trends Biochem Sci 31: 268-275.

Nichols A, Camps M, Gillieron C, Chabert C, Brunet A, Wilsbacher $\mathbf{J}$ et al. (2000). Substrate recognition domains within extracellular signal-regulated kinase mediate binding and catalytic activation of mitogen-activated protein kinase phosphatase-3. J Biol Chem 275: 24613-24621.

Pearson G, Robinson F, Beers Gibson T, Xu BE, Karandikar M, Berman K et al. (2001). Mitogen-activated protein (MAP) kinase pathways: regulation and physiological functions. Endocr Rev 22: 153-183.

Rigas JD, Hoff RH, Rice AE, Hengge AC, Denu JM. (2001). Transition state analysis and requirement of Asp-262 general acid/base catalyst for full activation of dualspecificity phosphatase MKP3 by extracellular regulated kinase. Biochemistry 40: 4398-4406.

Rohan PJ, Davis P, Moskaluk CA, Kearns M, Krutzsch H, Siebenlist U et al. (1993). PAC-1: a mitogen-induced nuclear protein tyrosine phosphatase. Science 259: 1763-1766.

Salojin KV, Owusu IB, Millerchip KA, Potter M, Platt KA, Oravecz T. (2006). Essential role of MAPK phosphatase-1 in the negative control of innate immune responses. J Immunol 176: 1899-1907.

Saxena M, Mustelin T. (2000). Extracellular signals and scores of phosphatases: all roads lead to MAP kinase. Semin Immunol 12: 387-396.

Slack DN, Seternes OM, Gabrielsen M, Keyse SM. (2001). Distinct binding determinants for ERK2/p38alpha and JNK map kinases mediate catalytic activation and substrate selectivity of map kinase phosphatase-1. J Biol Chem 276: 16491-16500.

Smith TG, Karlsson M, Lunn JS, Eblaghie MC, Keenan ID, Farrell ER et al. (2006). Negative feedback predominates over cross-regulation to control ERK MAPK activity in response to FGF signalling in embryos. FEBS Lett 580: 4242-4245.

Smith TG, Sweetman D, Patterson M, Keyse SM, Munsterberg A. (2005). Feedback interactions between MKP3 and ERK MAP kinase control scleraxis expression and the specification of rib progenitors in the developing chick somite. Development 132: 1305-1314.

Stewart AE, Dowd S, Keyse SM, McDonald NQ. (1999). Crystal structure of the MAPK phosphatase Pyst 1 catalytic domain and implications for regulated activation. Nat Struct Biol 6: 174-181.

Tanoue T, Adachi M, Moriguchi T, Nishida E. (2000). A conserved docking motif in MAP kinases common to substrates, activators and regulators. Nat Cell Biol 2: $110-116$.

Tanoue T, Maeda R, Adachi M, Nishida E. (2001a). Identification of a docking groove on ERK and p38 MAP kinases that regulates the specificity of docking interactions. EMBO J 20: 466-479.

Tanoue T, Moriguchi T, Nishida E. (1999). Molecular cloning and characterization of a novel dual specificity phosphatase, MKP-5. J Biol Chem 274: 19949-19956.

Tanoue T, Yamamoto T, Maeda R, Nishida E. (2001b). A Novel MAPK phosphatase MKP-7 acts preferentially on JNK/SAPK and p38 alpha and beta MAPKs. J Biol Chem 276: 26629-26639.

Tanoue T, Yamamoto T, Nishida E. (2002). Modular structure of a docking surface on MAPK phosphatases. J Biol Chem 277: 22942-22949. 
Theodosiou A, Ashworth A. (2002). MAP kinase phosphatases. Genome Biol 3: REVIEWS3009.

Tsang M, Maegawa S, Kiang A, Habas R, Weinberg E, Dawid IB. (2004). A role for MKP3 in axial patterning of the zebrafish embryo. Development 131: 2769-2779.

Volmat V, Pouyssegur J. (2001). Spatiotemporal regulation of the p42/p44 MAPK pathway. Biol Cell 93: 71-79.

Wada T, Penninger JM. (2004). Mitogen-activated protein kinases in apoptosis regulation. Oncogene 23: 2838-2849.

Willoughby EA, Collins MK. (2005). Dynamic interaction between the dual specificity phosphatase MKP7 and the JNK3 scaffold protein beta-arrestin 2. J Biol Chem 280: 25651-25658.

Willoughby EA, Perkins GR, Collins MK, Whitmarsh AJ. (2003). The JNK-interacting protein-1 scaffold protein targets MAPK phosphatase-7 to dephosphorylate JNK. J Biol Chem 278: 10731-10736.

Wu JJ, Roth RJ, Anderson EJ, Hong EG, Lee MK, Choi CS et al. (2006). Mice lacking MAP kinase phosphatase-1 have enhanced MAP kinase activity and resistance to dietinduced obesity. Cell Metab 4: 61-73.

Wu JJ, Zhang L, Bennett AM. (2005). The noncatalytic amino terminus of mitogen-activated protein kinase phosphatase 1 directs nuclear targeting and serum response element transcriptional regulation. Mol Cell Biol 25: 4792-4803.
Yin Y, Liu YX, Jin YJ, Hall EJ, Barrett JC. (2003). PAC1 phosphatase is a transcription target of p53 in signalling apoptosis and growth suppression. Nature 422: 527-531.

Zhang Q, Muller M, Chen CH, Zeng L, Farooq A, Zhou MM. (2005). New insights into the catalytic activation of the MAPK phosphatase PAC-1 induced by its substrate MAPK ERK2 binding. J Mol Biol 354: 777-788.

Zhang Y, Blattman JN, Kennedy NJ, Duong J, Nguyen T, Wang Y et al. (2004). Regulation of innate and adaptive immune responses by MAP kinase phosphatase 5. Nature 430: 793-797.

Zhao Q, Wang X, Nelin LD, Yao Y, Matta R, Manson ME et al. (2006). MAP kinase phosphatase 1 controls innate immune responses and suppresses endotoxic shock. $J$ Exp Med 203: 131-140.

Zhou B, Wu L, Shen K, Zhang J, Lawrence DS, Zhang ZY. (2001). Multiple regions of MAP kinase phosphatase 3 are involved in its recognition and activation by ERK2. $J$ Biol Chem 276: 6506-6515.

Zhou B, Zhang J, Liu S, Reddy S, Wang F, Zhang ZY. (2006). Mapping ERK2-MKP3 binding interfaces by hydrogen/ deuterium exchange mass spectrometry. J Biol Chem 281: 38834-38844.

Zhou B, Zhang ZY. (1999). Mechanism of mitogen-activated protein kinase phosphatase-3 activation by ERK2. J Biol Chem 274: 35526-35534. 